\title{
Fragment Deexcitation of Fission Induced by High Energy Nucleons
}

\author{
Oleg Grudzevich ${ }^{1, a}$ and Sergey Yavshits ${ }^{2}$ \\ 1 State Technical University, Obninsk, Russia \\ 2 Khlopin Radium Institute,St. Petersburg, Russia
}

\begin{abstract}
The KRIF library of the neutron-, proton- and gamma-spectra emitted by the nuclei excited up to $500 \mathrm{MeV}$ is presented. The KRIF contains information for about 2000 emitters which are the fragments of the ten targets fission induced by the nucleons with the energies up to $3 \mathrm{GeV}$.
\end{abstract}

\section{Introduction}

It is known the fission is the equilibrium process. Before the compound nucleus formation the equilibration of composite system has to be realized. We used two noncompound stage in computing of nucleon escaping before compound state forming. The intranuclear cascade (INC) model was used to describe nucleon escape and the distribution of energy between the reaction participants. The results of INC are the Y(ZA,E,ph) yields as a function of the excitation energy $\mathrm{E}$, exciton configuration ph and nucleus ZA. This 3D-matrix is the input data for the 2nd stage of the system going to equilibrium modeling.

The multi-particle preequilibrium (MCP) model [1] developed was exploited to get the distribution of the nuclei in different equilibrium excitation energies The verification of INC+MCP reliability was done by the comparison of the calculated nucleon spectra with the available experimental data for the all $(20-160 \mathrm{MeV})$ projectile energies and targets $(A=20-240)$.

The final stage of the all nuclear reactions (even for high energy projectiles) is the decay of equilibrated (compound) nuclei by particles, gamma-emission and fission. The statistical model (STA) of nuclear in Hauser-Feshbach formalism was used for all calculations of this reaction stage. The INC, MCP and STA approaches were combined as MCFx code [2]. To check the MCFx results we used the measured neutron multiplicities and fission cross sections additionally to mentioned above.

\section{Fission fragment decay}

The fission fragments are of course at the excited states. We believe that the decay of these nuclei starts after there complete acceleration. In this case it is ease to extract the excitation energies from the information on the neutron multiplicities [3]. The values of the mean fragment energies $<U>$ are presented in fig. 1 in comparison with the

\footnotetext{
a e-mail: grudzevich@iate.obninsk.ru
}

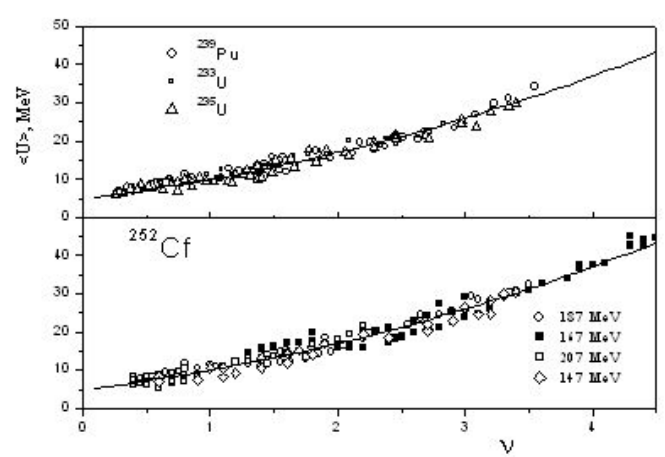

Fig. 1. Mean excitation energies of fission fragments as the function of the neutron multiplicity for different fissile systems: thermal neutron fission of ${ }^{233,235} \mathrm{U},{ }^{239} \mathrm{Pu}$ (top) and spontaneous fission of ${ }^{252} \mathrm{Cf}$ for the different TKE.

prompt neutron multiplicities $v$ of the fission of ${ }^{233,235} \mathrm{U}$, ${ }^{239} \mathrm{Pu}$ by thermal neutrons and spontaneous fission of ${ }^{(252) \mathrm{Cf}}$ for different TKE. The dependence can be describes by the formula

$$
<U>=5+4 v+v^{2}
$$

In the fission induced by high energy nucleons the fission neutron multiplicity value is $10-25$ units so total fragments excitation will be $100-500 \mathrm{MeV}$ (see fig.8). The data on excitation energies of each fragments or even sum complementary fragment energy can be used for the computing of all FF decay characteristics: $n$ - and g-spectra, mean spins, isomeric ratios, multiplicities, etc.

\subsection{Neutron emission}

The examples of the experimental neutron multiplicity descriptions as a function of FF total kinetic energies are presented in fig.2. One can conclude the coincidence of the values for all fragments is rather good without fitting. The multiplicity values changes from 1 to 6 units as a kinetic

This is an Open Access article distributed under the terms of the Creative Commons Attribution-Noncommercial License, which permits unrestricted use, distribution, and reproduction in any noncommercial medium, provided the original work is properly cited. 


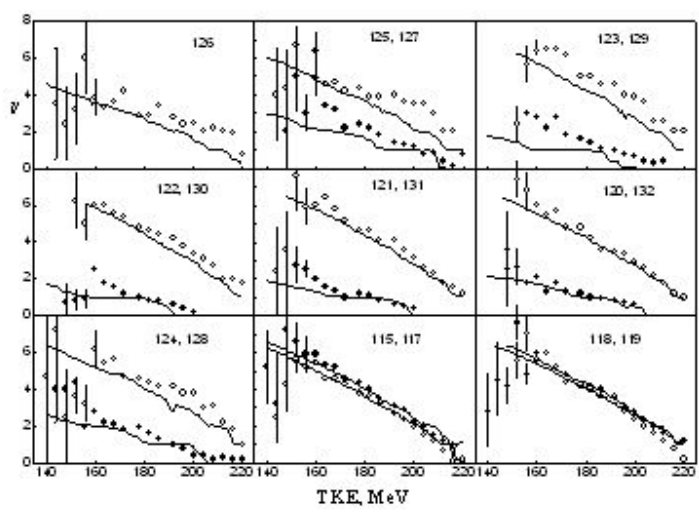

Fig. 2. Multiplicities of neutrons emitted by fragments of ${ }^{252} \mathrm{Cf}$ spontaneous fission versus total kinetic energy. Symbols are experimental data[4], the curves show the calculation results.

energy decreases. It means the excitation energy changes from $10 \mathrm{MeV}$ to $60 \mathrm{MeV}$ (see fig.1). The neutron spectra from thermal neutron induced fission of ${ }^{235} \mathrm{U}$ are shown in the fig.3. The measured energy distributions of prompt neutrons for different fragment masses $(\mathrm{A}=88-148)$ are reproduced satisfactory. The discrepancies take place for the fragment with $\mathrm{A}=154$ where the experimental error bars of [5] are large too. The examples of the fig.2-3 show typical situations of the available experimental neutron data description by the method [3].

\subsection{Gamma emission}

The $\gamma$ - emission from the fission fragments can be described by the statistical model of nuclear reactions rather successfully too in the case when one has information on the excitation energies of the fission fragments. The comparison of experimental data on the prompt $\gamma$ 's from fission fragments with the calculation results is presented in fig.4. Despite of the good agreement some large underestimation of soft energy rays takes place in this case. More clear this fact can be seen in fig. 5 where $\gamma$-multiplicities as a function of the fragment masses is shown. The calculated numbers of photons for the fragments with $\mathrm{A}=105-115$ and $\mathrm{A}=145$ 155 are smaller then experimental by the factor of 2 . This discrepancy can be originated from the method features.

We used a gamma-cascade model of nucleus deexcitation with no individual rotational bands. For the fragments mentioned (with large deformations) the $\gamma$-transitions between the members of the bands can do considerable contribution to the total multiplicity value. The energies of these photons are $0.5-1.0 \mathrm{MeV}$ and they will be placed at the beginning of the spectrum of fig. 4 and this contribution to calculated $\gamma$-multiplicity will improve the experimental data description.

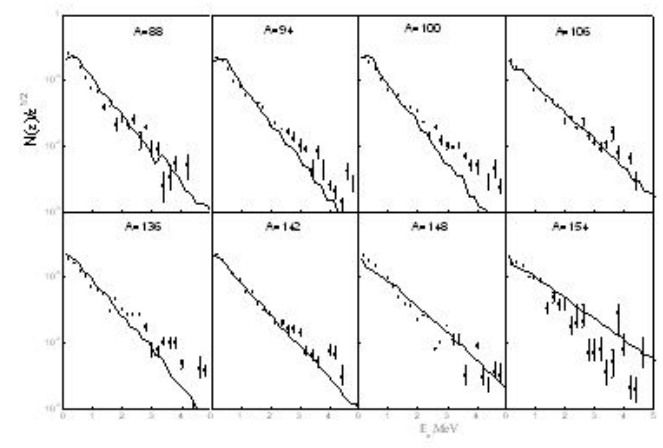

Fig. 3. Prompt neutron spectra of $235 \mathrm{U}$ thermal neutron fission. The symbols are the data of [5], the lines show the calculation results.

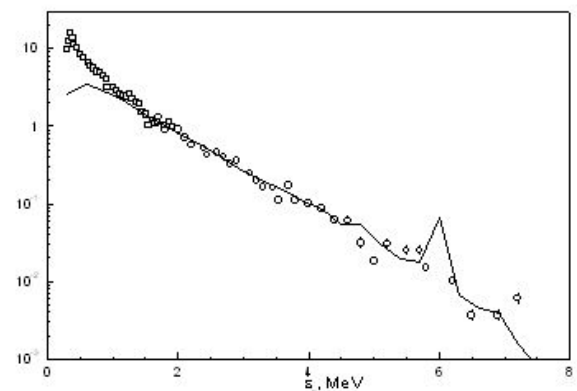

Fig. 4. Gamma multiplicities of fragments produced in thermal neutron fission of ${ }^{235} \mathrm{U}$. The symbols are experimental data [6], the curve is the results of calculations.

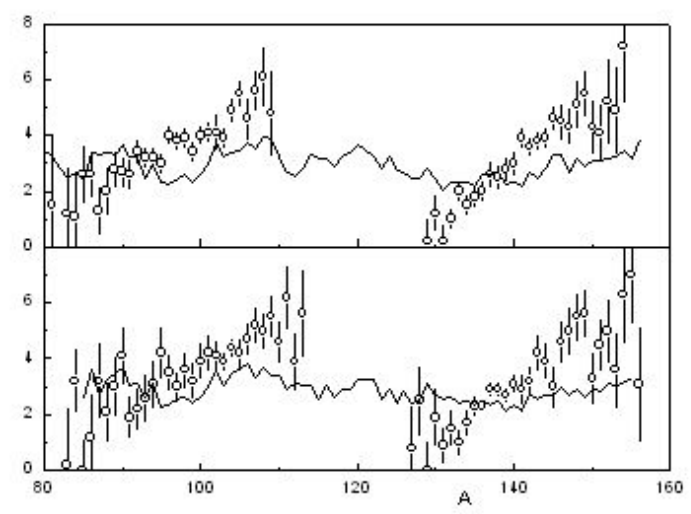

Fig. 5. Multiplicities of photons from fragments of thermal neutron fission of ${ }^{235} \mathrm{U}$ (top) and ${ }^{239} \mathrm{Pu}$ (bottom). Experimental data of [7], the curves are results of calculations. 


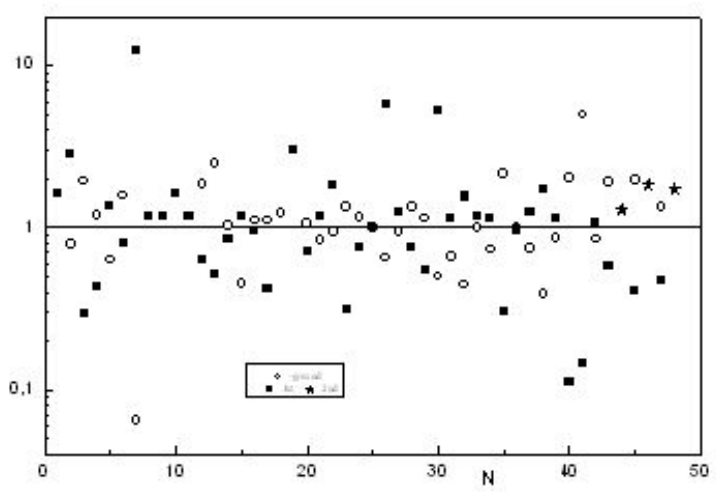

Fig. 6. The ratio of experimental [8] and calculated isomeric ratios of the independent yields of fragments originated from thermal neutron fission of ${ }^{235} \mathrm{U}$. The open circles are for ground states, closed squares show 1st metastable states, stars present high-spin states.

\subsection{Level independent yields}

The data on the independent yields of the fission fragments are very important information both for the nuclear physics theory and for the applications, e.g. the complete files of nuclear data. The isomeric ratios (IRIY) of independent yields (the ratio of fixed energy level population to the sum population) have the same meaning. According to the mechanism of isomer population, the IRIY of $(\mathrm{Z}, \mathrm{A}) \mathrm{nu}-$ cleus can be calculated by

$$
R=\frac{\sum Y_{i}(Z, A+i)<P_{i} r_{i}>}{\sum Y_{i}(Z, A+i)<P_{i}>},
$$

where $\mathrm{Y}_{i}$ is the independent yield of $(\mathrm{Z}, \mathrm{A}+\mathrm{i})$ fragment which emits i-neutrons, $\mathrm{P}_{i}$ is the probability of i-neutron emission, and $\mathrm{r}_{i}$ is the isomeric ratio for this case.The summing is for $\mathrm{i}=0-5$.

The experimental data on IRIY are available for ${ }^{235} \mathrm{U}$ fission by the thermal neutrons [8] of 48 isomeric pairs of the fragments in the range $A=79-148$. The characteristics of these levels are not unique - as a rule, the difference between the spins are a few units of $\hbar$. Only three highspin levels of the ${ }^{120,122,130}$ In isotopes (the stars in fig.6) have $\Delta \mathrm{J} \geq 7 \hbar$. The comparison of experimental [8] and calculated values of IRIY is shown in fig.6. It can be seen the model of these characteristics computing is reliable for the prediction of the data needed.

\section{KRIF library content}

The zero version of the KRIF-0 library of the data on the fission fragment emission characteristics was generated to do the investigations discussed above. The library covers the limited range of excitation energies (up to $200 \mathrm{MeV}$ ) and the nuclei of a rather narrow range. The example of KRIF-0 data is shown in fig.7 for the fission of ${ }^{235} \mathrm{U}$ target. The neutron number energy dependence behavior of

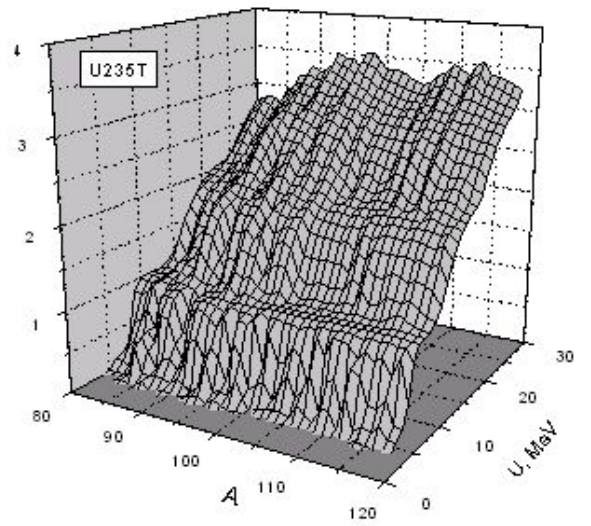

Fig. 7. The neutron multiplicity dependences on the excitation energy of the fragments of ${ }^{235} \mathrm{U}$ fission.

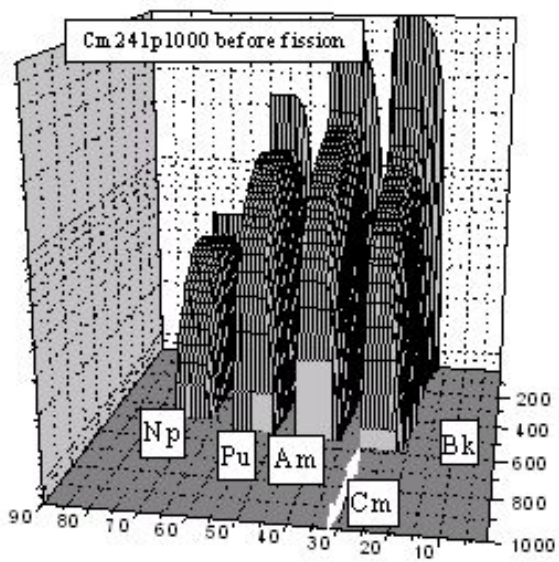

Fig. 8. Distribution of equilibrated nuclei on excitation energies in the proton interaction with ${ }^{241} \mathrm{Cm}$ target at the $1000 \mathrm{MeV}$ entrance energy.

the fragment with different mass number is understandable. One can see the step like view of the neutron emission probability.

An actual task of this work is the scope of all possible fission fragments of ten target-nuclei $(\mathrm{Pb}-\mathrm{Cm})$ for entrance channel energies from $20 \mathrm{MeV}$ up to $3 \mathrm{GeV}$. The analysis of the nuclear excitations after nonequilibrium nucleon escape, the equilibrium (fissioning) nuclei can be at the energies, making approximately 50 percents of the energy of a nucleon beam (fig. 8). It means, that the fission fragments can have the excitation energy the same order, say, for 1 $\mathrm{GeV}$ projectile energy the sum energy of complementary fragments will make $500 \mathrm{MeV}$.

The candidates for inclusion into the library were determined for all targets simultaneously. The analysis of experimental data about the fission fragments mass yields for all areas of the entrance channel energies and for all dia- 


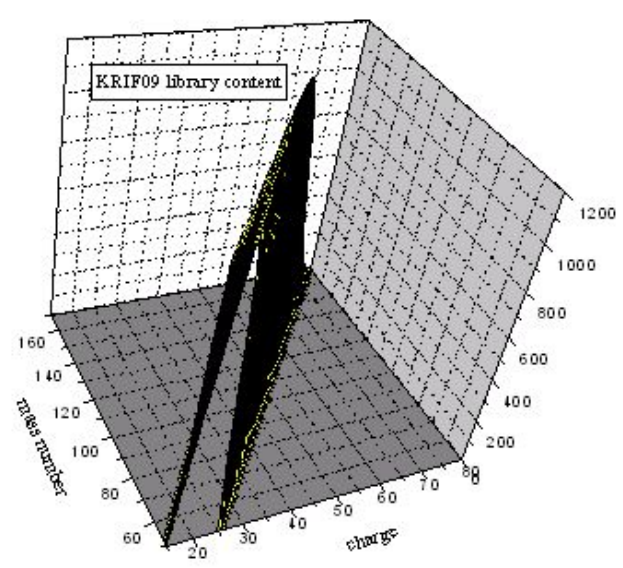

Fig. 9. The frequencies of the given nucleus realizations as the FF of different targets from ${ }^{208} \mathrm{~Pb}$ to ${ }^{241} \mathrm{Cm}$.

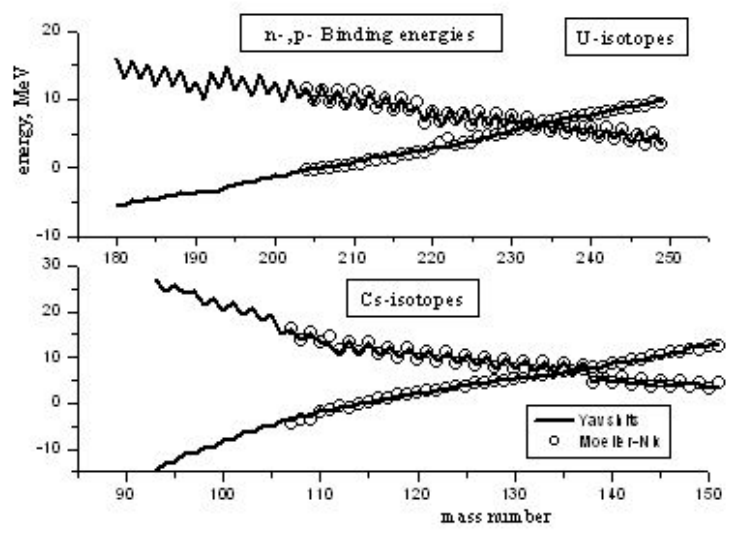

Fig. 10. Neutron and proton binding energies of uranium (top) and cesium (bottom) isotopes. The circles are RIPL-2 data [9], the curves show finite range method calculations.

pasons the target masses shows, that the mass numbers of $\mathrm{FF}$ are in a range from $\mathrm{A}=50$ to $\mathrm{A}=170$. The range is extended on \pm 10 mass units in order to avoid the missing or not taken into account some exotic nuclei. The number of protons which are escaped at the noncompound stages is equal to 4-5 units. The fragment charges were determined from a condition of an unchanged charge distribution (invariance of charge density) at the fission processes. The deviation from the most probable charge $Z_{p}$ in distribution was accepted as three charge units, that obviously is more than expected value. The list of the FF nuclei for library KRIF09 is generated.

The representation about the frequencies of the given nucleus realizations as the FF of different targets from ${ }^{208} \mathrm{~Pb}$ to ${ }^{241} \mathrm{Cm}$ is possible to get from fig. 9 . One can see the unexpected small width of the distribution (number of cases of realization of the fixed fragment) on the charges. In procedure of the FF list preparation for the separate fissile system the broad charge distribution is incorporated, and the fissioning nuclei differ on a charge from initial com- posite system (projectile plus target) on four - five charge units. As it can be expected, the significant part of the total number of the nuclei is made by the proton exceeded ones. The calculations of these nuclei deexcitation can have the specific features because of small proton binding energies, therefore we analyzed these FF especially.

The binding energies file of RIPL-2 library [9] contains not enough long isotopic chains, e.g. for cesium element (fig.10). In the case when one of Cs-isotopes, say 135 or 137 , is realized as a fission fragment at $300 \mathrm{MeV}$ excitation this nucleus may decay by 25-30 neuron emission. The final nucleus will be out of the range covered by RIPL-2 data (see fig.10). The calculated by the finite range method nucleon binding energies needed for the input data for the more long isotopic chains are shown in fig.10 in comparison with the RIPL-2 data.

The calculations of the nucleon and gamma-emission from the nuclei excited up to hundreds mega-electron-volts were carried out for the current version of the library KRIF09. We accepted the greatest value of the energy as $500 \mathrm{MeV}$.

\section{Conclusion}

The KRIF09 library of fission fragments of the heavy nucleus targets $\left({ }^{208} \mathrm{~Pb}-{ }^{241} \mathrm{Cm}\right)$ induced by the nucleons with $20 \mathrm{MeV}-3 \mathrm{GeV}$ energies is discussed. The KRIF contains the information on 2000 nuclei excited up to $500 \mathrm{MeV}$. The work was performed under ISTC Project 3751.

\section{References}

1. Grudzevich O. et al. Oxford University Press, Radiation Protection Dosimetry, Volume 26, 1-4, (2007), 101-103.

2. Yavshits S. et al. In Proc. of the Int. Conf. on Nucl. Data for Sci. and Tech., Tsukubo, Japan, (2001) pp. 104-107 .

3. Grudzevich O. Physics of Atomic nuclei, Volume 64, 9, (2001), pp. 1643-1659.

4. C. Budtz-Jorgensen and H. H. Knitter, Nucl. Phys., Volume A490, (1988), p. 307.

5. K.Nishio, I.Kimura and Y.Nakagome, ISINN-7, Dubna, (1997), p. 325.

6. V.S.Stavinskii, Physics of Nuclear Fission (Gosatomizdat, Moscow 1963).

7. F.Pleasonston, Nucl. Phys., Volume A213, (1973), p. 413.

8. G.Rudstam, Proc. of aa Spec. Meeting on Fiss. Prod. Nucl. Data, Tokai, Japan, 1992, p.271.

9. Reference Input Parameter Library, htpp://wwwnds.iaea.org/ripl 